\title{
Endovascular embolisation strategies for pulmonary arteriovenous malformations
}

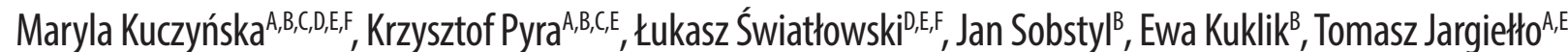

Department of Interventional Radiology and Neuroradiology, Medical University of Lublin, Poland

\section{Abstract}

Purpose: To assess the immediate efficacy of distinct embolisation devices in the endovascular treatment of pulmonary arteriovenous malformations as well as to identify and analyse the possible determinants of the technical success of the procedure.

\begin{abstract}
Material and methods: Prospective analysis of 16 consecutive patients was carried out, who underwent transcatheter endovascular embolisation therapy for pulmonary arteriovenous malformations between 2005 and 2017. Pre- and post-procedural angiography studies were implemented to confirm the diagnosis and to evaluate the technical success defined as the complete occlusion of the feeding artery. Embolisation devices - coils, microcoils, occluders, or combination of the above - were used. All the patients were advised to conduct a follow-up computed tomography evaluation 12 months after the procedure.
\end{abstract}

Results: A total of 40 pulmonary arteriovenous malformations (PAVMs) were observed and embolised. The immediate technical success was achieved in all $(n=40 ; 100 \%)$ treated PAVMs, as confirmed by the post-procedural angiographic result. The statistical analysis revealed no significant impact of the number of PAVMs per patient $(p>0.05)$, their angioarchitecture $(p>0.05)$, localisation within the lung $(p>0.05)$ or particular lobe $(p>0.05)$, and the selection of embolisation device $(p>0.05)$ on the procedural success rates. The procedure-related complication rate was equal to $6.25 \%$.

Conclusions: The immediate success rate of the transcatheter PAVM embolisation reached $100 \%$ in this study. The statistical model of logistic regression revealed no significant impact of the number of PAVMs per patient, their angioarchitecture, localisation, and distribution pattern, as well as device selection, on the immediate technical success of the procedure.

Key words: arteriovenous fistula, therapeutic embolisation, Amplatzer occluder, pulmonary AVM.

\section{Introduction}

Pulmonary arteriovenous malformation (PAVM) is a rare clinical occurrence with a reported incidence rate of 1 in 100,000 individuals. The estimated female-to-male ratio ranges from $1.5: 1$ to $1.8: 1$. PAVM is a pathologic direct communication between the feeding pulmonary artery branch and the dysplastic, aneurysmal pulmonary vein that results in right-to-left shunt. The shunt is predis- posing for the development of paradoxical embolisation - a major cause of PAVM-related mortality [1]. The malformations can be classified as simple (approximately 80\%) with a unique feeding vessel and singular draining vein, or complex [1,2]. A large majority of the pulmonary AVMs (50-83\%) are multiple and located within the inferior lobes [1,2].

As much as $90 \%$ of the lesions are expected to be associated with the hereditary haemorrhagic telangiectasia

\footnotetext{
Correspondence address:

Maryla Kuczyńska, MD, Department of Interventional Radiology and Neuroradiology, Medical University of Lublin, 8 Jaczewskiego St. , 20-954 Lublin, Poland, e-mail:maryla.kuczynska@gmail.com
}

Authors' contribution:

A Study design · B Data collection · C Statistical analysis · D Data interpretation · E Manuscript preparation · F Literature search · G Funds collection 
(HHT), known as the Osler-Weber-Rendu syndrome, which is an autosomal dominant disease [1,2]. This implies careful screening for the disease in individuals with PAVMs, who have not been previously diagnosed with Osler-Weber-Rendu disease [1].

The condition may be clinically silent or manifest as either respiratory or neurological disorders $[1,2]$. A high percentage of occult visceral malformations (PAVMs in $53 \%$ cases) was detected in the study by Giordano et al. performed in a group of 35 paediatric patients with genetically-confirmed diagnosis of HHT [3]. In addition, a retrospective cohort study aiming to assess the prevalence of silent brain infarcts (SBI) among patients with HHT revealed that the SBI rate was higher in HHT patients with PAVMs when compared to that of the general population and HHT patients without pulmonary malformations [4]. According to Pollak et al., approximately 59\% of patients with PAVMs will experience dyspnoea or exercise intolerance, $3 \%$ hemothorax, $18 \%$ transient ischaemic attacks, $33 \%$ ischaemic stroke, and $23 \%$ cerebral abscess [5]. Interestingly, as reported by Shovlin et al., incidence rates of the severe neurological complications are independent of both the magnitude of the right-to-left shunt and the diameter of the largest feeding artery (FA; determinants of severity of PAVMs) [6]. Conversely, the diffuse, bilateral pattern of the disease seems to be associated with higher mortality rates [7].

Considering the above-mentioned risks associated with PAVMs, an attentive and meticulous diagnostic approach seems to be of the utmost importance. However, the task is particularly challenging, as the study results indicate that as much as 14 to $80 \%$ of individuals with PAVMs may be diagnosed accidentally, by incidental investigations or PAVM screening programmes in families with HHT $[2,6]$. In addition, merely $20 \%$ of patients had PAVMs diagnosed by the imaging modalities or oxygenation measurements performed due to respiratory symptoms [6]. It is therefore essential to distinguish individuals with high risk of PAVMs, i.e. those with clinical features suggestive of the disorder or with suspected or known diagnosis of the HHT, and initiate diagnostic evaluation in this group $[8,9]$. Assessing the presence and magnitude of the right-to-left shunt by means of transthoracic contrast echocardiography (TTCE) is advised as the initial screening approach. Positive screening results should be confirmed with unenhanced chest computed tomography (CT) with thin-cut reconstructions [8]. Patients qualified for embolotherapy based on prior CT evaluation are referred for a catheter pulmonary angiography, which remains the gold standard in the diagnosis of PAVM. The modality is applied with the primary purpose of diagnosis confirmation and therapeutic management [9]. In addition, it enables visualisation of the detailed angioarchitecture of even the smallest PAVMs, which contribute to device selection process.
Although no results of the randomised, controlled trial have been published yet, the collective observational data suggest that transcatheter embolisation is an effective and safe procedure that contributes to decreased PAVM-associated morbidity and mortality rates $[1,6]$. In addition, there is general agreement that the role of surgical interventions should be restricted to the management of emergency situations including haemorrhages $[1,8]$. Major indications for the embolotherapy comprise: the prevention of the life-threatening neurological (i.e. ischaemic stroke, cerebral abscess) and pulmonary haemorrhagic complications, as well as improvement in exercise tolerance $[1,6,10]$. Recent publications stress the importance of PAVM treatment in both symptomatic and asymptomatic patients amenable to embolisation, even if the feeding artery diameter is less than $3 \mathrm{~mm}$. In such cases, the procedure was proven to serve as protection against bacterial spread and paradoxical embolisation $[1,5,8]$. The primary procedure's goal of PAVM embolisation is the occlusion of the feeding vessel, which may be accompanied by the closure of the venous aneurysmal sac to achieve improved outcomes in complex malformations $[1,11,12]$.

Because the complete eradication of PAVMs in people with HHT is impossible due to the persistence of previously treated lesions and formation of new lesions, a longterm follow-up by means of CT of the thorax is highly recommended $[1,8]$. As assessed during consecutive CT examinations, the permanent success is defined by the following criteria: disappearance or marked reduction (30-70\% shrinkage) of the PAVM remnant and/or lack of enhancement of the AVP sac and adjacent draining vein in contrast-enhanced scans [13].

The authors aimed to assess the immediate efficacy of distinct embolisation devices in the endovascular treatment of pulmonary arteriovenous malformations, as well as to identify and analyse the possible determinants of the technical success of the procedure.

\section{Material and methods}

We performed a prospective analysis of 16 consecutive patients who underwent transcatheter endovascular embolisation therapy for pulmonary arteriovenous malformations between 2005 and 2017.

The inclusion criteria comprised the presence of PAVM confirmed by diagnostic imaging (including pre-procedural angiography) and either one of the following: presence of clinical symptoms (e.g. pulmonary haemorrhage, hypoxaemia), feeding artery diameter $>3 \mathrm{~mm}$, or rapid enlargement of the lesion confirmed by imaging studies. The exclusion criteria included generally accepted contraindications to embolotherapy, i.e. severe chronic kidney disease without availability of dialysis and every pulmonary hypertension. 
Patient demographics (age, sex) and symptomatological information were obtained from the hospital documentation. Diagnostic, procedural, and equipment details were obtained from the local radiological information system.

Embolisation procedures were performed under local anaesthesia, using the Siemens Artis angiography system setup. Each patient was administered with a $5000 \mathrm{IU}$ bolus of heparin immediately prior to embolisation, to prevent clot formation and the risk of cerebral embolisation. Pre- and post-procedural angiography studies were implemented to confirm the diagnosis and evaluate technical success defined as the complete occlusion of the feeding artery with no evidence of residual blood flow through the PAVM, respectively. In each case, the transfemoral venous approach with 5-6 F, $65 \mathrm{~cm}$-long vascular sheath was executed to reach the main pulmonary artery, with subsequent selective embolisation of the PAVM feeding branch with a $5 \mathrm{~F}$ Vert or Cobra catheter. An additional coaxial 2.7 F micro-catheter (Progreat ${ }^{\oplus}$, Terumo Interventional Systems) was used if applicable. The embolisation device (pushable 0.035 " coil, 0.018 " microcoil, Amplatzer ${ }^{\text {Tn }}$ occluder, or a combination of the above) was selected based on the size of the feeding vessel and angioarchitecture of the lesion, as visualised during pre-procedural angiography.

All the patients were advised to conduct a follow-up CT evaluation 12 months after the procedure or upon symptom recurrence.

The statistical analysis was performed using Statistica v. 12.0 (StatSoft Poland). Numerical data were presented as mean \pm standard deviation and median values, whereas categorical data were given in absolute frequencies and percentages. Logistic regression was applied to investigate the possible impact of the particular variables (number and localisation of PAVMs, embolisation device) on the immediate technical outcome of the procedure. A value of $p<0.05$ indicated statistical significance.

\section{Results}

There were 16 patients included in the study: 10 women and 6 men (female-to-male ratio $1.67: 1$ ) aged between 22 and 77 years (mean age $44.63 \pm 17.47$ years, median $=40$ years $)$. One patient $(n=1 ; 6.25 \%)$ had a confirmed diagnosis of HHT. A total of 40 PAVMs were observed. In eight (50\%) patients the lesions were multiple and localised bilaterally (Figure 1), in seven (43.75\%) patients they were single and unilateral (Figure 2), and in one (6.25\%) patient they were multiple and unilateral. A large majority of PAVMs $(n=31 ; 77.5 \%)$ occupied inferior lobes of the lungs. Only three (7.5\%) PAVMs had complex angioarchitecture. Detailed angioarchitecture and distribution characteristics of the malformations are listed in Table 1. Fifteen out of 16 patients required a single-step procedure. One patient $(n=1 ; 6.25 \%)$ required a two-stage procedure
(Figure 3) due to numerous PAVMs localised bilaterally. The technical success was assessed per stage in this case. The majority ( $n=16 ; 40 \%$ ) of PAVMs were embolised using a combination of 0.038 " pushable coils with 0.018 " microcoils, indicating the need for dense filling of the coil scaffold in order to achieve complete immediate technical success. In addition, only a single need to support the use of Amplatzer ${ }^{\text {Tw }}$ vascular plug with additional coil implantation shows the great potential of this device to serve as a solitary, effective measure of simple PAVM treatment. Treatment approach and device selection characteristics are presented in Table 2.

The procedure-related complication rate was $6.25 \%$ ( $n=1$ patient). Blood-tinged sputum was observed in one patient during coil embolisation of a complex PAVM, which was probably caused by punctuation of one of the supplying arterial branches by the tip of the guide wire. The condition was self-limiting, and a complete resolution of symptoms was achieved within 15 minutes. Control erect chest radiograph and specialist consultation confirmed no pathology.

Immediate technical success was achieved in all $(n=40$; $100 \%)$ treated PAVMs, as confirmed by the post-procedural angiographic result. The statistical analysis revealed no significant impact of the number of PAVMs per patient $(p>0.05)$, their angioarchitecture $(p>0.05)$, localisation within the lung $(p>0.05)$ or particular lobe $(p>0.05)$, and the selection of embolisation device $(p>0.05)$ on the procedural success rates.

\section{Discussion}

The reported female-to-male ratio (1.67: 1) of PAVM incidence among the studied population is in accordance with previously published data [1]. The statistics concerning distribution of the lesions within the lungs $(77.5 \%$ PAVMs localised in the inferior lobes), their angioarchitecture (92.5\% of simple lesions), and multiplicity (56.25\% patients with multiple PAVMs) are consistent with the literature $[1,2,12]$. However, the reported prevalence of HHT among the studied population (6.25\%) is far from the generally accepted estimations $[1,2]$. This indicates the further necessity for thorough genetic and clinical examination of patients who meet at least some of the Curaçao diagnostic criteria for HHT $[1,6]$. Nonetheless, study results presented by Shovlin et al. do not support extensive screening in asymptomatic children [6].

The immediate technical success rate, defined as the occlusion of FA visible on angiogram obtained just after embolisation, which was adapted for the purpose of this study, reached $100 \%(n=16)$. These results are also consistent with the previously published data, which indicate the success rates between $80 \%$ and $100 \%$ regardless of the embolisation material $[5,10,14-16]$. The statistical analysis proved the results to be independent of the number of PAVMs embolised, their angioarchitecture, localisation, 

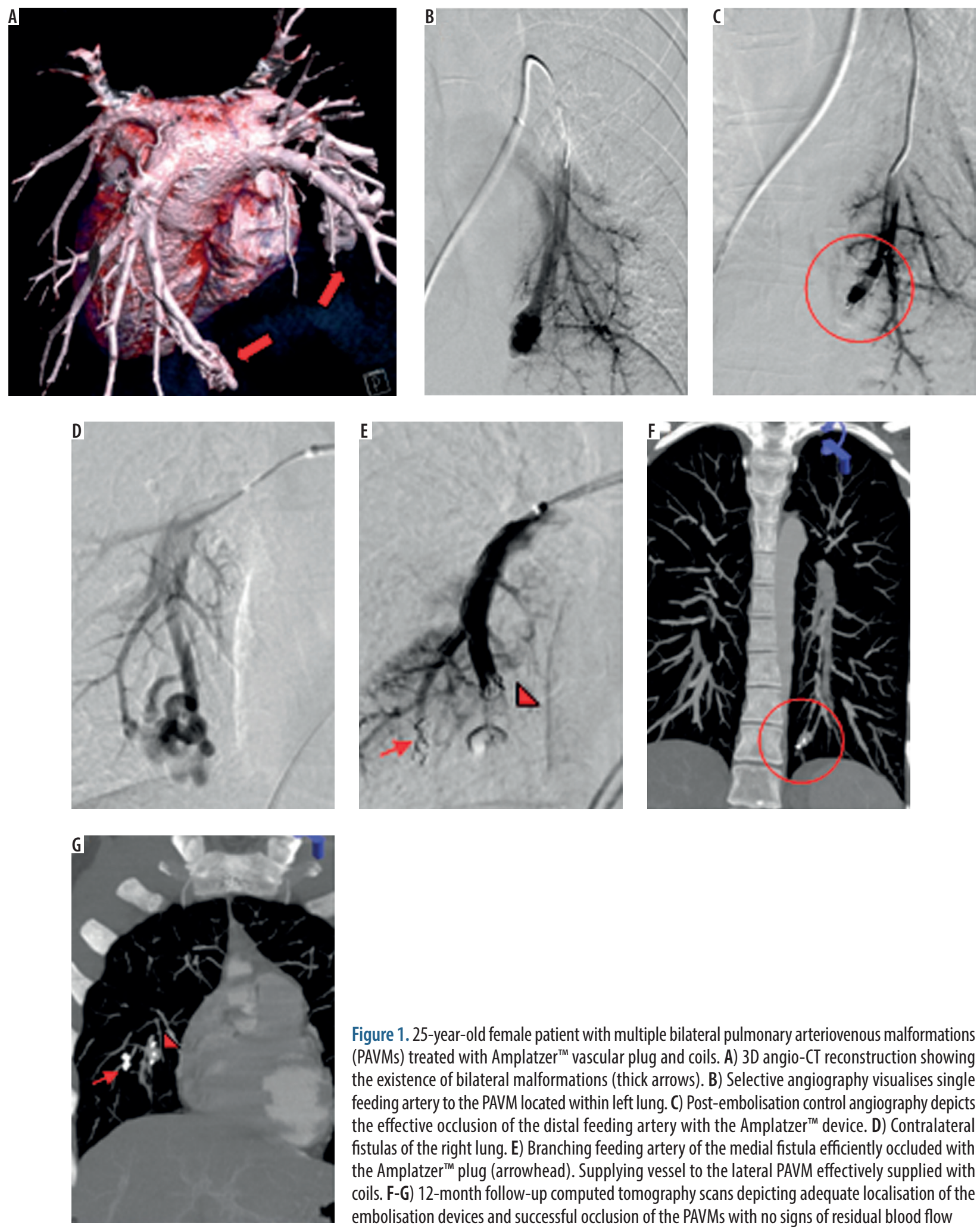

Figure 1.25-year-old female patient with multiple bilateral pulmonary arteriovenous malformations (PAVMs) treated with Amplatzer ${ }^{\mathrm{TM}}$ vascular plug and coils. A) 3D angio-CT reconstruction showing the existence of bilateral malformations (thick arrows). B) Selective angiography visualises single feeding artery to the PAVM located within left lung. C) Post-embolisation control angiography depicts the effective occlusion of the distal feeding artery with the Amplatzer ${ }^{\mathrm{TM}}$ device. D) Contralateral fistulas of the right lung. E) Branching feeding artery of the medial fistula efficiently occluded with the Amplatzer ${ }^{\mathrm{TM}}$ plug (arrowhead). Supplying vessel to the lateral PAVM effectively supplied with coils. F-G) 12-month follow-up computed tomography scans depicting adequate localisation of the embolisation devices and successful occlusion of the PAVMs with no signs of residual blood flow

and distribution pattern as well as device selection. These results are also consistent with the previously published data, which indicate success rates between $80 \%$ and $100 \%$ regardless of the embolisation material [5,10,14-16].

The selection of the embolisation device is mostly determined by the angioarchitecture and feeding artery diameter of the lesion [1]. In general, PAVMs with FA diameter $>3 \mathrm{~mm}$ may be supplied with the Amplatzer ${ }^{\mathrm{mm}}$ vascular occluder (St. Jude Medical). The device allows for safe and convenient positioning and redeployment under fluoroscopic guidance, to achieve the best possible direct occlusion of the supplying vessel. The mechanism of action of the vascular plug can be compared to previously described detachable-balloons, which were withdrawn from the market. The reported effectiveness of the procedures performed using the Amplatzer ${ }^{\mathrm{rm}}$ device are 

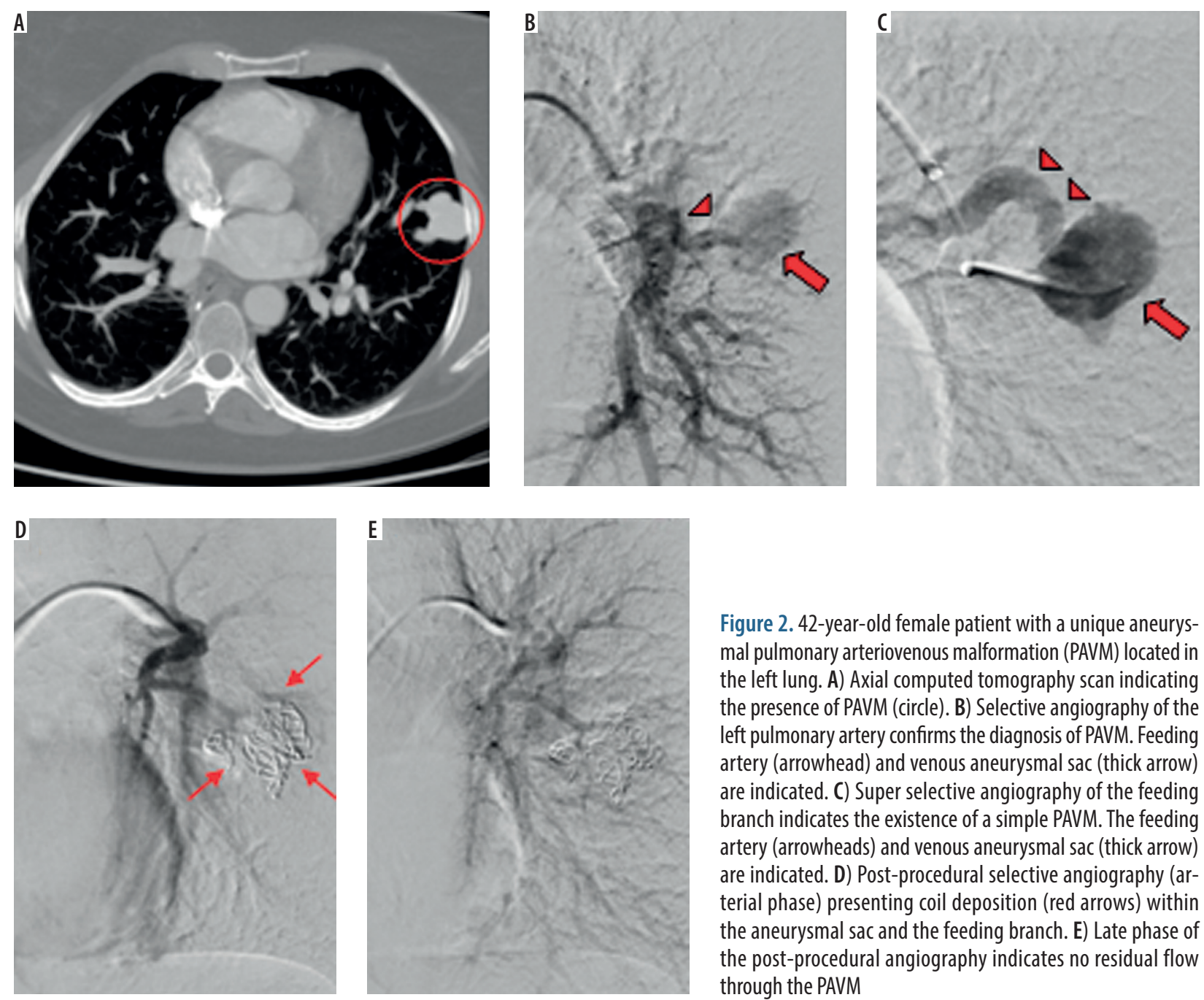

Figure 2. 42-year-old female patient with a unique aneurysmal pulmonary arteriovenous malformation (PAVM) located in the left lung. A) Axial computed tomography scan indicating the presence of PAVM (circle). B) Selective angiography of the left pulmonary artery confirms the diagnosis of PAVM. Feeding artery (arrowhead) and venous aneurysmal sac (thick arrow) are indicated. C) Super selective angiography of the feeding branch indicates the existence of a simple PAVM. The feeding artery (arrowheads) and venous aneurysmal sac (thick arrow) are indicated. D) Post-procedural selective angiography (arterial phase) presenting coil deposition (red arrows) within the aneurysmal sac and the feeding branch. E) Late phase of the post-procedural angiography indicates no residual flow through the PAVM

Table 1. Detailed localisation and angioarchitecture of pulmonary arteriovenous malformations (PAVMs)

\begin{tabular}{|l|c|c|c|c|c|}
\hline \multirow{3}{*}{ Lung } & Lobe & Total $n$ PAVMs & \multirow{2}{*}{$n$ PAVMs } & \multicolumn{2}{|c|}{ Angioarchitecture } \\
\cline { 5 - 6 } & & & & $n$ simple & $n$ complex $^{*}$ \\
\hline \multirow{3}{*}{ Right } & Superior & \multirow{2}{*}{$17(42.5 \%)$} & 3 & 3 & n/a \\
\cline { 2 - 4 } & Middle & & 2 & 2 & n/a \\
\cline { 2 - 4 } & Inferior & & $12(30 \%)$ & 10 & 2 \\
\cline { 2 - 4 } & Superior & \multirow{2}{*}{$23(57.5 \%)$} & 4 & 3 & n/a \\
\cline { 2 - 4 } & Inferior & & $19(47.5 \%)$ & 19 & $3(7.5 \%)$ \\
\hline
\end{tabular}

*Complex angioarchitecture refers to PAVMs with feeding artery to draining vein ratio other than 1:1

$n$ - number of lesions; $n / a-$ not applicable

promising, with an estimated $100 \%$ immediate technical success rates and $0-7 \%$ reperfusion rates $[1,14,15,17]$. Embolisation experience with 0.035 " pushable coils has been broadly published. Unlike the vascular occluder, fibre coverage of the coils evokes indirect vessel occlusion due to activation of the platelet aggregation $[1,5,18]$. It has been learned over time that dense packing of the coils is essential to achieve a durable result, especially because a large diameter of the FA has been associated with ele- vated risk for reperfusion $[5,14]$. This in turn, may form a niche for the use of micro delivery equipment along with platinum detachable microcoils. The combined use of both the 0.035 " and 0.018 " devices may lead to increased packing density and, therefore, contribute to reduced distant reperfusion rates. In addition, micro equipment may be employed during embolisation of distal pulmonary arterial branches that are not amenable for catheterisation with standard 4-5 F guiding catheters, and thus constitute 

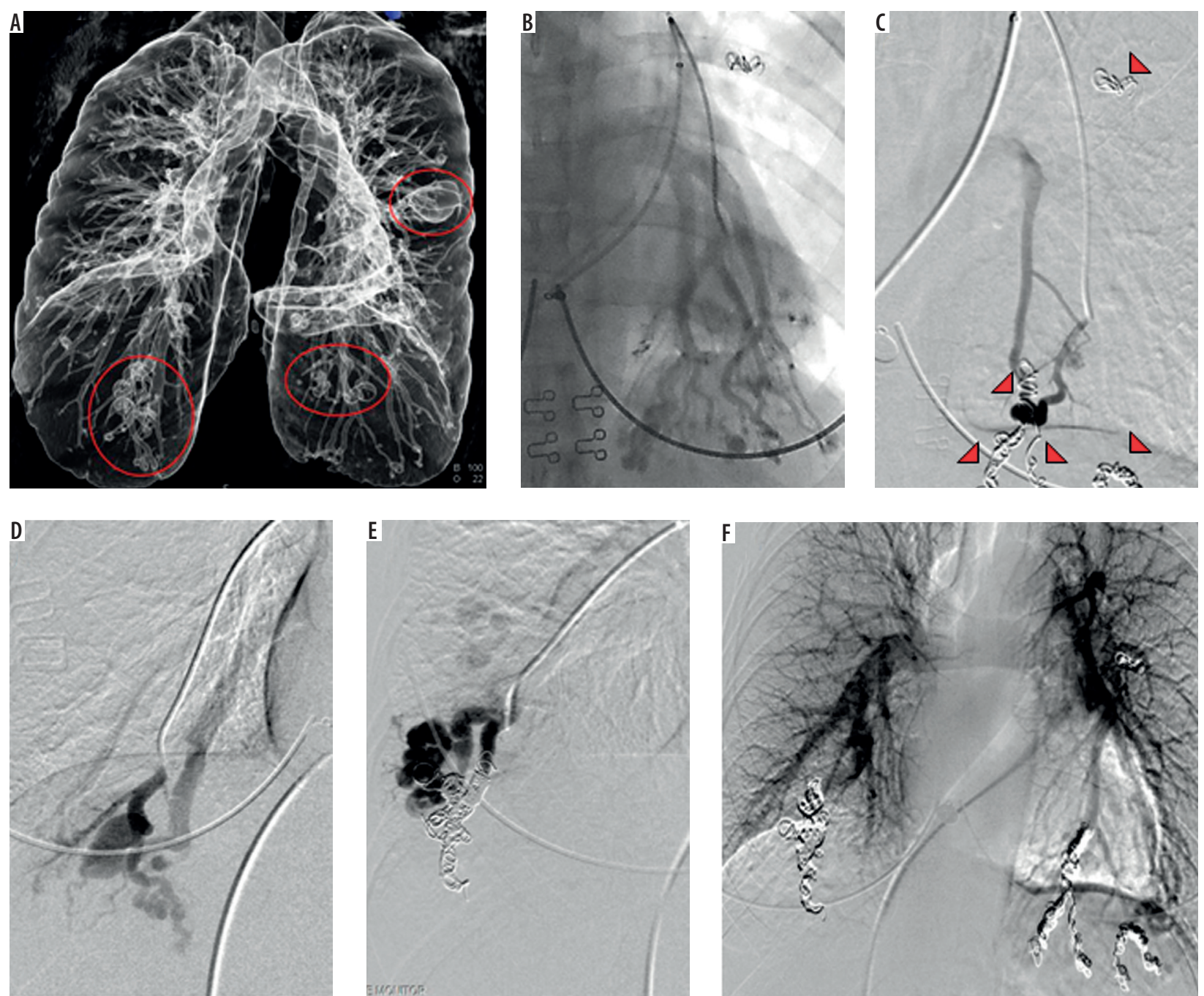

Figure 3. 36-year-old female patient diagnosed with hereditary haemorrhagic telangiectasia (HHT) and multiple bilateral pulmonary arteriovenous malformations (PAVMs). A) 3D angio-CT reconstruction shows the existence of numerous lesions in both lungs (red circles). B) First stage of the procedure. Multiple PAVMs localised in the lower lobe of the left lung. C) Pre-embolisation angiography of a high-flow simple malformation. Coils visible inside the occluded feeding arteries of the already treated malformations (arrowheads). D-E) Second stage of embolisation. Successful occlusion of the lesions of the right lung. F) Control angiography performed after second stage of embolisation indicates effective occlusion of all treated PAVMs

Table 2. Device/treatment selection with respect to pulmonary arteriovenous malformation angioarchitecture

\begin{tabular}{|c|c|c|c|c|c|c|}
\hline & $\begin{array}{l}\text { Pushable } 0.038^{\prime \prime} \\
\text { coils }\end{array}$ & $\begin{array}{c}0.018^{\prime \prime} \\
\text { microcoils }\end{array}$ & $\begin{array}{c}0.038^{\prime \prime} \text { pushable coils + } \\
0.018^{\prime \prime} \text { microcoils }\end{array}$ & $\begin{array}{l}\text { Amplatzer }^{\mathrm{TM}} \\
\text { occluder }\end{array}$ & $\begin{array}{l}\text { Amplatzer }{ }^{\mathrm{TM}} \text { occluder } \\
0.038^{\prime \prime}+\text { pushable coils }\end{array}$ & \\
\hline$n$ simple & 8 & 6 & 15 & 7 & 1 & 37 \\
\hline$n$ complex & $\mathrm{n} / \mathrm{a}$ & 1 & 1 & $\mathrm{n} / \mathrm{a}$ & 1 & 3 \\
\hline Total $n$ PAVMs & 8 & 7 & 16 & 7 & 2 & 40 \\
\hline
\end{tabular}

$n$ - number of lesions, $n / a-$ not applicable

a promising treatment option for patients with diffuse pattern of small PAVMs. However, such observations have not been supported by White, who advocates that the use of microcoils adds to the cost and complexity of the embolisation procedure $[11,19]$. Nonetheless, if one decides to perform a procedure with the use of the microcoils, caution should be applied to avoid non-target embolisation, particularly in high-flow malformations [12].

It should be stressed, however, that the encouraging initial results do not translate into long-term efficacy of the embolisation procedure [14]. Persistence of the PAVM is defined as continued perfusion through the lesion after its endovascular treatment and may occur in either of the two mechanisms: recanalisation or reperfusion. Recanalisation is associated with reestablishment of the flow through the previously occluded feeding artery, and it accounts for as much as $90 \%$ of persistent PAVMs. Conversely, reperfusion develops as a result of the pulmonary-to-pulmonary or systemic-to-pulmonary connections $[5,14,16]$. According to the literature, there is a substantial difference in PAVM 
persistence between embolisation with use of coils and embolisation with use of vascular plugs [14,16]. Reported experience with the Amplatzer ${ }^{\text {tw }}$ occluder has shown low reperfusion rates of $0-7 \%$, perhaps with even better results when the plug was combined with the use of coils [13]. Persistence rates after initial treatment with the use of coils alone were as high as $8-25 \%[14,16]$. Nonetheless, no randomised controlled trials have been executed until present to compare the efficacy of selected types of embolisation coils. The authors of this publication express their hope that a current clinical trial, aimed to compare reperfusion rates observed after the use of Nester coil and Interlock Fibered IDC Occlusion System, will elucidate this matter to some extent [20].

A recent study by Martin et al. indicates that both primary and recurrent recanalisation rates are influenced also by the post-embolisation antithrombotic therapy $(\mathrm{OR}=15.21,95 \% \mathrm{CI}: 1.54-150.13, p=0.020$ and $\mathrm{OR}=6.40,95 \% \mathrm{CI}: 1.31-31.26, p=0.02$, respectively). These observations pose a new challenge for the clinicians to consider when prescribing antithrombotic agents to patients with HHT [21].

The observed complication rates in our cohort were equal to $6.25 \%(n=1)$. The self-limiting haemoptysis occurred during the procedure and was probably caused by punctuation of a small arterial branch with the tip of the guide wire. This implies that embolisation is a safe and effective procedure for pulmonary arteriovenous malformations.

There were certain limitations to this study. Firstly, the study population ( $n=16)$ was small, which might have influenced the outcome of the analysis. Secondly, it was impossible to conduct a multivariate statistical analysis to assess the possible relationship between the immediate technical success and the diameter of the feeding artery and selected equipment, due to incomplete hospital documentation concerning the exact type and size of the chosen devices. In addition, despite the fact that the patients were advised to undergo a follow-up CT scan 12 months after the embolisation, the scans could have not be included in the analysis of long-term results of the procedures due to the following issues: (i) CT evaluation scans of $11(68.75 \%)$ subjects are still pending because the embolisation was performed in less than 12 months; (ii) follow-up CT scans $(n=3)$ performed in regional centres differ in terms of slice thickness; and (iii) some patients $(n=2)$ were lost to follow-up.

Based on the above observations, the authors of this publication are convinced that individuals with HHT should be referred to a specialised centre, where extensive, targeted care may be executed. It is also of the utmost importance to introduce definite follow-up criteria that would include exact CT scan parameters to enable reliable comparative studies regarding the long-term efficacy of the particular embolisation devices.

\section{Conclusions}

The immediate success rate of the transcatheter PAVM embolisation reached $100 \%$ in this study, regardless of the device selection. The statistical model of logistic regression revealed no significant impact of the number of PAVMs per patient, their angioarchitecture, localisation, and distribution pattern as well as device selection on the immediate technical success of the procedure.

\section{Acknowledgements}

This work was made possible by the collaboration of the staff and faculty at the Department of Interventional Radiology and Neuroradiology, Medical University of Lublin, Poland.

\section{Conflict of interest}

The authors report no conflict of interest.

\section{References}

1. Hsu CC, Kwan GN, Evans-Barns H, et al. Embolisation for pulmonary arteriovenous malformation. Cochrane Database Syst Rev 2018; 1: CD008017.

2. White RI Jr, Lynch-Nyhan A, Terry P, et al. Pulmonary arteriovenous malformations: techniques and long-term outcome of embolotherapy. Radiology 1988; 169: 663-669.

3. Giordano P, Nigro A, Lenato GM, et al. Screening for children from families with Rendu-Osler-Weber disease: from geneticist to clinician. J Thromb Haemost 2006; 4: 1237-1245.

4. Brinjikji W, Nasr DM, Wood CP, et al. Pulmonary Arteriovenous Malformations Are Associated with Silent Brain Infarcts in Hereditary Hemorrhagic Telangiectasia Patients. Cerebrovasc Dis 2017; 44: 179-185.
5. Pollak JS, Saluja S, Thabet A, et al. Clinical and anatomic outcomes after embolotherapy of pulmonary arteriovenous malformations. J Vasc Interv Radiol 2006; 17: 35-44.

6. Shovlin CL, Jackson JE, Bamford KB, et al. Primary determinants of ischaemic stroke/brain abscess risks are independent of severity of pulmonary arteriovenous malformations in hereditary haemorrhagic telangiectasia. Thorax 2008; 63: 259-266.

7. Pierucci P, Murphy J, Henderson KJ, et al. New definition and natural history of patients with diffuse pulmonary arteriovenous malformations: twenty-seven-year experience. Chest 2008; 133: 653-661.

8. Faughnan ME, Palda VA, Garcia-Tsao G, et al. International guidelines for the diagnosis and management of hereditary haemorrhagic telangiectasia. J Med Genet 2011; 48: 73-87. 
9. Gossage Jr, Kanj G. Pulmonary arteriovenous malformations. A state of the art review. Am J Respir Crit Care Med 1998; 158: 643-661.

10. Mager JJ, Overtoom TT, Blauw H, et al. Embolotherapy of pulmonary arteriovenous malformations: long-term results in $112 \mathrm{pa}-$ tients. J Vasc Interv Radiol 2004; 15: 451-456.

11. White RI Jr. Pulmonary arteriovenous malformations: how do I embolize? Tech Vasc Interv Radiol 2007; 10: 283-290.

12. Hundt W, Kalinowski M, Kiessling A, et al. Novel approach to complex pulmonary arteriovenous malformation embolization using detachable coils and Amplatzer vascular plugs. Eur J Radiol 2012; 81: 732-738.

13. Remy-Jardin M, Dumont P, Brillet PY, et al. Pulmonary arteriovenous malformations treated with embolotherapy: helical CT evaluation of long-term effectiveness after 2-21-year follow-up. Radiology 2006; 239: 576-585.

14. Pollak JS, White RI Jr. Distal cross-sectional occlusion is the "key" to treating pulmonary arteriovenous malformations. J Vasc Interv Radiol 2012; 23: 1578-1580.

15. Rabellino M, Serra M, Peralta O, et al. Early experience with the AMPLATZER vascular plug IV for the occlusion of pulmonary arteriovenous malformations. J Vasc Interv Radiol 2014; 25: 1333-1337.
16. Abdel Aal AK, Eason J, Moawad S. Persistent Pulmonary Arteriovenous Malformations: Percutaneous Embolotherapy. Curr Probl Diagn Radiol 2017; https://doi.org/10.1067/j.cpradiol.2017.09.006.

17. Tau N, Atar E, Mei-Zahav M. Amplatzer Vascular Plugs Versus Coils for Embolization of Pulmonary Arteriovenous Malformations in Patients with Hereditary Hemorrhagic Telangiectasia. Cardiovasc Intervent Radiol 2016; 39: 1110-1114.

18. Borrero CG, Zajko AB. Pulmonary arteriovenous malformations: clinical features, diagnosis, and treatment. J Radiol Nurs 2006; 25: 33-37.

19. White RI Jr, Pollak JS, Picus D. Are Guglielmi detachable coils necessary for treating pulmonary arteriovenous malformations? Radiology 2003; 226: 599-600.

20. NCT01856842. Reperfusion of pulmonary arteriovenous malformations after embolotherapy. A randomized trial of Interlock ${ }^{\mathrm{m}}$ Fibered IDC $^{\mathrm{mw}}$ Occlusion System vs. Nester Coils. Available at: https://clinicaltrials.gov/ct2/show/NCT01856842 (Accessed: 04 February 2018).

21. Martin JL, Faughnan ME, Prabhudesai V. Antithrombotic Use Predicts Recanalization of Embolized Pulmonary Arteriovenous Malformations in Hereditary Hemorrhagic Telangiectasia. Can Assoc Radiol J 2017; 68: 463-467. 